\title{
On the definition and calculation of a generalised McIlwain parameter
}

\author{
J. Pilchowski ${ }^{1}$, A. Kopp ${ }^{2}$, K. Herbst ${ }^{2}$, and B. Heber ${ }^{2}$ \\ ${ }^{1}$ Geophysical Institute, 903 Koyukuk Drive, Univ. of Alaska, Fairbanks, AK 99775-7320, USA \\ ${ }^{2}$ Inst. für Experimentelle und Angewandte Physik, Christian-Albrechts-Univ. zu Kiel, Leibnizstraße 11, 24118 Kiel, Germany
}

Received: 3 December 2009 - Revised: 3 February 2010 - Accepted: 10 February 2010 - Published: 22 April 2010

\begin{abstract}
The $L$ parameter, which indicates the distance where a magnetic field line crosses the equatorial plane, is defined only for an aligned magnetic dipole field. For a realistic planetary magnetic field, however, neither a definition nor a method to calculate this parameter are available so far. We therefore extent the definition of the McIlwain parameter for an arbitrary planetary magnetic field and numerically calculate it for the actual geomagnetic field. In order to do so, we first calculate the Earth's magnetic field for 2008 with the IGRF model. To motivate a proper definition for a general $L$ parameter, each component of this field will be illustrated and discussed. In a second step, we present four possible definitions for the $L$ parameter and discuss their properties and differences with respect to the question in how far they reflect the field geometry. We contrast our method with the traditional derivation of the $L$ parameter employing numerical simulations of the cut-off rigidities of energetic particles and an empirical relation between the latter and $\mathrm{L}$.
\end{abstract}

\section{Introduction and basic facts}

Already in the middle of the nineteenth century, Carl Friedrich Gauss demonstrated that the Earth's magnetic field B can be derived from the sum of contributions resulting from external and internal source regions. If electric currents $\mathbf{j}$ can be neglected within the region of interest, i.e.

$$
\nabla \times \mathbf{B}=\mu_{0} \mathbf{j}=0,
$$

where $\mu_{0}$ is the permeability of vacuum, $\mathbf{B}$ can be written as the gradient of a scalar potential $\Gamma$, (e.g Gauss, 1836):

$\mathbf{B}=-\nabla \Gamma$.

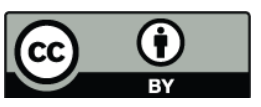

Correspondence to: J. Pilchowski (jpilchowski@alaska.edu)
Because of the divergence-free condition for $\mathbf{B}, \Gamma$ has to be the solution of the Laplace equation

$\Delta \Gamma=0$.

In general, the scalar potential $\Gamma$ can be expanded into a series of spherical harmonics. Using spherical coordinates $(r$, $\vartheta, \varphi$ ), this expansion reads (e.g. Connerney, 1993):

$$
\begin{aligned}
& \Gamma(r, \vartheta, \varphi)= \\
& r_{0} \sum_{n=1}^{\infty}\left\{\left(\frac{r}{r_{0}}\right)^{n} T_{n}^{(\mathrm{ext})}(\vartheta, \varphi)+\left(\frac{r_{0}}{r}\right)^{n+1} T_{n}^{(\mathrm{int})}(\vartheta, \varphi)\right\}
\end{aligned}
$$

with $r_{0}$ representing the Earth's radius $\left(r_{0}=6371 \mathrm{~km}\right)$, while $T_{n}^{\text {(ext) }}(\vartheta, \varphi)$ and $T_{n}^{\text {(int) }}(\vartheta, \varphi)$ denote the external and internal contributions of source regions, respectively, which can be written as

$$
\begin{aligned}
& T_{n}^{(\mathrm{ext})}(\vartheta, \varphi)= \\
& \sum_{m=0}^{n}\left\{P_{n}^{m}(\cos \vartheta)\left(G_{n}^{m} \cos (m \varphi)+H_{n}^{m} \sin (m \varphi)\right)\right\}, \\
& T_{n}^{\text {(int) }}(\vartheta, \varphi)= \\
& \sum_{m=0}^{n}\left\{P_{n}^{m}(\cos \vartheta)\left(g_{n}^{m} \cos (m \varphi)+h_{n}^{m} \sin (m \varphi)\right)\right\} .
\end{aligned}
$$

$G_{n}^{m}, \quad H_{n}^{m}, \quad$ as $\quad$ well as $g_{n}^{m}$ and $h_{n}^{m}$ are the (Schmidt-)coefficients, and $P_{n}^{m}$ are the Schmidt-Legendre functions of orders $n$ and $m$. They are defined as:

$$
P_{n}^{m}(x)=N_{n m} \sqrt{(1-x)^{m}} \frac{d^{m} P_{n}(x)}{d x^{m}}
$$

with $N_{n m}$ and $P_{n}$ being the normalisation factors and the Legendre polynomials of order $n$, respectively:

$N_{n m}= \begin{cases}1 & m=0 \\ \sqrt{\frac{2(n-m) !}{(n+m) !}} & m \neq 0\end{cases}$

Published by Copernicus Publications on behalf of the Arbeitsgemeinschaft Extraterrestrische Forschung e.V. 


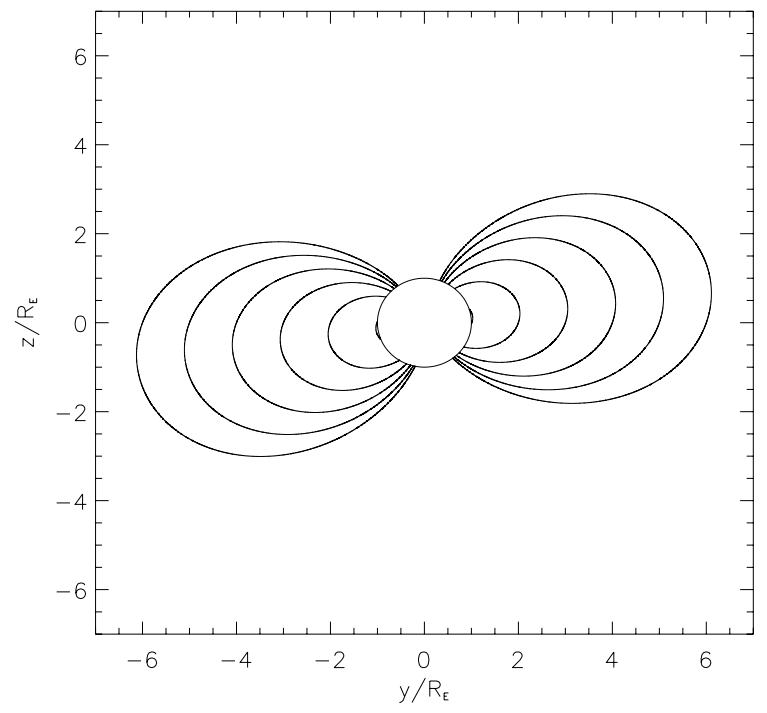

Fig. 1. Field lines of the Earth's magnetic field calculated with the IGRF model for 2008 in cartesian coordinates with $\mathrm{z}$ being parallel to the magnetic field axis.

$P_{n}(x)=\frac{1}{2^{n} n !} \frac{d^{n}\left(x^{2}-1\right)^{n}}{d x^{n}}$

A first approximation of the Earth's magnetic field is a tilted dipole field, where the position of the dipole centre and the position of Earth's centre coincide (geocentric dipole approximation). This approximation is useful in regions, where the influence of the Solar wind can be neglected (cf. Sect. 2) or for the academic case in which the field is viewed from larger distances in absence of the Solar wind. At a given position $\mathbf{r}$, this field can be written as

$\mathbf{B}(\mathbf{r})=\frac{3(\mathbf{m} \cdot \mathbf{r})}{r^{5}} \mathbf{r}-\frac{\mathbf{m}}{r^{3}}$,

where $\mathbf{m}^{1}$ is the magnetic moment and $r=|\mathbf{r}|$. This expression simply results from the lowest-order term of expansion (4), i.e. $n=1$ and $m=0$, considering only internal sources, leading to

$\Gamma(r, \vartheta, \varphi)=r_{0}^{3}\left(\begin{array}{l}g_{1}^{1} \\ h_{1}^{1} \\ g_{1}^{0}\end{array}\right) \cdot \frac{1}{r^{3}}\left(\begin{array}{l}x \\ y \\ z\end{array}\right)=\frac{\mathbf{m} \cdot \mathbf{r}}{r^{3}}$.

Considering $\mathbf{m}$ to be tilted by the angle $\Theta$ with respect to the axis of rotation (the z-axis) and by the angle $\Phi$ around it, the magnetic moment can be expressed as

$\mathbf{m}=m_{0}\left(\begin{array}{c}\sin \Theta \cos \Phi \\ \sin \Theta \sin \Phi \\ \cos \Theta\end{array}\right)=r_{0}^{3}\left(\begin{array}{l}g_{1}^{1} \\ h_{1}^{1} \\ g_{1}^{0}\end{array}\right)$.

\footnotetext{
${ }^{1}$ not to be confused with the index $m$ in Eqs. (5-10)
}

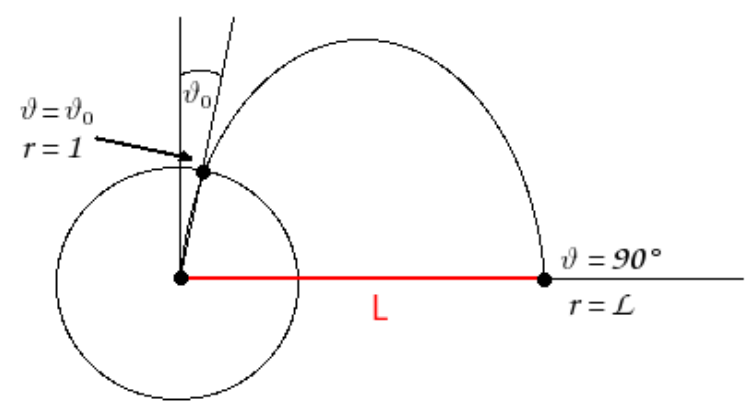

Fig. 2. Illustration of the $L$ parameter depending on the geographical co-latitude $\vartheta$ for an aligned dipole.

These angles and the strength of the dipole can be expressed by means of the expansion coefficients as:

$$
\begin{aligned}
m_{0} & =r_{0}^{3} \sqrt{\left(g_{1}^{1}\right)^{2}+\left(h_{1}^{1}\right)^{2}+\left(g_{1}^{0}\right)^{2}} \\
\Theta & =\arccos \left(g_{1}^{0} / \sqrt{\left(g_{1}^{1}\right)^{2}+\left(h_{1}^{1}\right)^{2}+\left(g_{1}^{0}\right)^{2}}\right), \\
\Phi & =\arctan \left(h_{1}^{1}, g_{1}^{1}\right) .
\end{aligned}
$$

One accurate model for the Earth's magnetic field currently available is the International Geomagnetic Reference Field ${ }^{2}$, but as the values for $g_{1}^{1}, h_{1}^{1}$, and $g_{1}^{0}$ represent only the first terms of the expansion, the field is better represented by a tilted dipole with its centre being displaced from Earth's centre by the vector $\mathbf{r}_{q}$ (eccentric dipole approximation). Other magnetic field models for instance are POMME 3 and POMME 4 (POtsdam Magnetic Model of the Earth) ${ }^{3}$ that are based on satellite data.

Today, the geographical latitude and longitude of the point where the axis of an aligned dipole intersects the Earth's surface in the Southern Hemisphere are given as $\Theta=168.6^{\circ}$ (78.6 degree south) and $\Phi=109.9^{\circ}$ (or degree east), respectively, with a magnetic field strength of $|\mathbf{B}|=30760 \mathrm{nT}$. The values for the dipole shift are $\left|\mathbf{r}_{q}\right|=0.0725$ Earth radii, $\Theta_{q}=71.7^{\circ}$ (18.3 degree north) and $\Phi_{q}=147.8^{\circ}$ (or degree east $)^{4}$. The lowest order terms of the IGRF expansion result in $|\mathbf{B}|=30037 \mathrm{nT}, \Theta=169.7^{\circ}$ and $\Phi=108.2^{\circ}$.

A few field lines near the equator of the complete magnetic field as represented by the IGRF model for 2008 is shown in Fig. 1 within the first six Earth radii.

\section{Traditional computation of the $L$ parameter}

The McIlwain or L parameter (McIlwain, 1961) is defined only for an aligned (i.e. untilted) dipole field. At a given

\footnotetext{
${ }^{2}$ http://www.ngdc.noaa.gov/IAGA/vmod/igrf.html

${ }^{3} \mathrm{http}: / /$ www.geomag.us/models/pomme3.html

${ }^{4} \mathrm{http} / / / \mathrm{nssdc} . \mathrm{gsfc}$. nasa.gov/planetary/factsheet/earthfact.html
} 


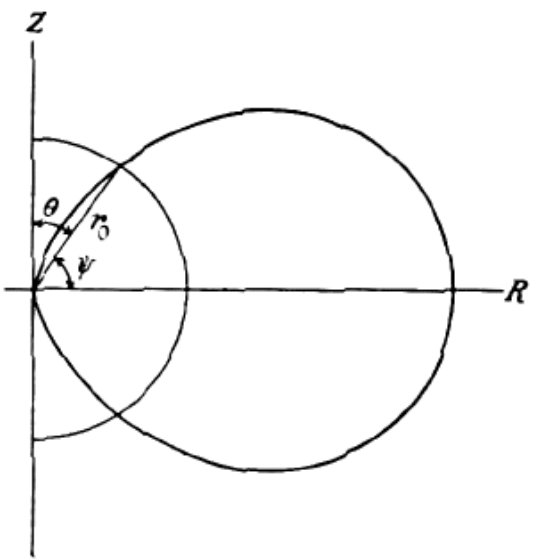

Fig. 3. Illustration of a Størmer orbit and the definition of the angle $\vartheta=90^{\circ}-\psi$. The value $r_{0}$ represents the Earth's radius (Størmer, 1955).

co-latitude $\vartheta_{0}$ it can be calculated by the simple relation (cf. Stern (1969) for a derivation)

$$
L=\frac{1}{\sin ^{2}\left(\vartheta_{0}\right)} .
$$

As illustrated in Fig. 2, $L$ is the distance in Earth radii, at which a magnetic field line penetrating the Earth's surface at the co-latitude $\vartheta_{0}$ crosses the equatorial plane. Because of the fact that the $L$ parameter is neither defined for a general planetary field, nor can be calculated even for the definition above, it must be computed indirectly. The traditional method to do so employs the Størmer orbits in auroral latitudes. Carl Størmer's work dealt with charged particles penetrating into the Earth's magnetic field at high latitudes, leading eventually to the formation of auroral lights. Therefore, he calculated the so-called forbidden and allowed particle trajectories of charged particles moving towards the Earth (Størmer, 1955). The origin of charged particles detected in the Earth's magnetic field can be established by (nowadays numerically) retracing the particles' trajectories back into the interplanetary space, rather than to compute the orbits towards the Earth. Forbidden orbits are those remaining close to Earth or returning to it. The allowed ones are those along which the particles can actually escape. Such an Størmer orbit, where the distance, $r$, parameterised by the angle $\vartheta$ with $r_{0}$ representing the Earth's radius (cf. Fig. 3) is given by Størmer (1955) as

$r=C_{s t} \cdot \frac{\sin ^{2} \vartheta}{1+\sqrt{1+\sin ^{3} \vartheta}}$

with $r$ being given in Earth radii and the Størmer constant $C_{s t}=\sqrt{m / P}$, which contains the magnetic moment $m=|\mathbf{m}|$ of the Earth and the rigidity (momentum per charge) $P$ of the particles.

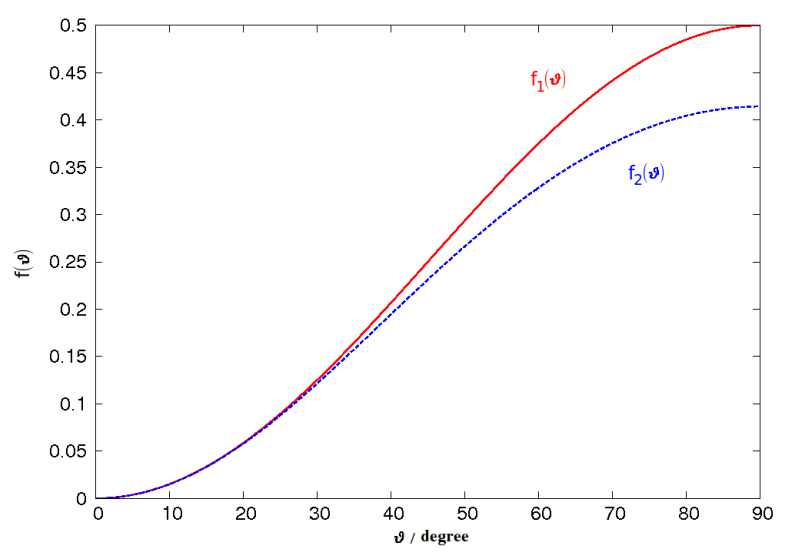

Fig. 4. Comparison of the functions $f_{1}(\vartheta)=\sin ^{2} \vartheta / 2$ and $f_{2}(\vartheta)=\sin ^{2}(\vartheta) /\left(1+\sqrt{1+\sin ^{3} \vartheta}\right)$ with $\vartheta$ being the co-latitude.

Because Størmer was only interested in high latitudes, i.e. regions of small values of $\vartheta$, he could neglect the $\sin ^{3} \vartheta$ term in the denominator, leading to

$r^{2}=\frac{m}{4 P} \cdot \sin ^{4} \vartheta$,

giving a relation between the rigitity $P$ that denotes a particle's momentum per unit charge, and the $L$ parameter, if the latter is inserted according to Eq. (13) and evaluated on the Earth's surface, i.e. $r=r_{0}$, leading to

$P=K \cdot \frac{1}{L^{2}}$,

with $K=m /\left(4 r_{0}\right)=14.81 \mathrm{GV}$.

A more realistic dependency was given by Shea and Smart (1986) by using a least-square fit of simulated and measured data. They found:

$P=\tilde{K} \cdot \frac{1}{L^{q}}$

with the slightly different values $\tilde{K}=14.823 \mathrm{GV}$ and $q=2.0311$.

The traditional approach to compute the $L$ parameter consists of performing numerical simulations or employing measurements in order to obtain the maximum rigidity, at which a particle can hit the Earth's surface at a given latitude on an allowed orbit, the so-called cut-off rigidity, and relate the latter to the respective $L$ parameter by means of Eq. (17). In order to calculate the $L$ parameter along a trajectory or for a part of the Earth's surface, the cut-off rigidity has, thus, to be known for every point along it.

In addition to the numerical or observational effort to obtain the cut-off rigidities, we would like to point out here that Eq. (17) is used for the entire surface of the Earth, but makes use of an approximation (cf. Eq. 15) being valid only in auroral latitudes. 

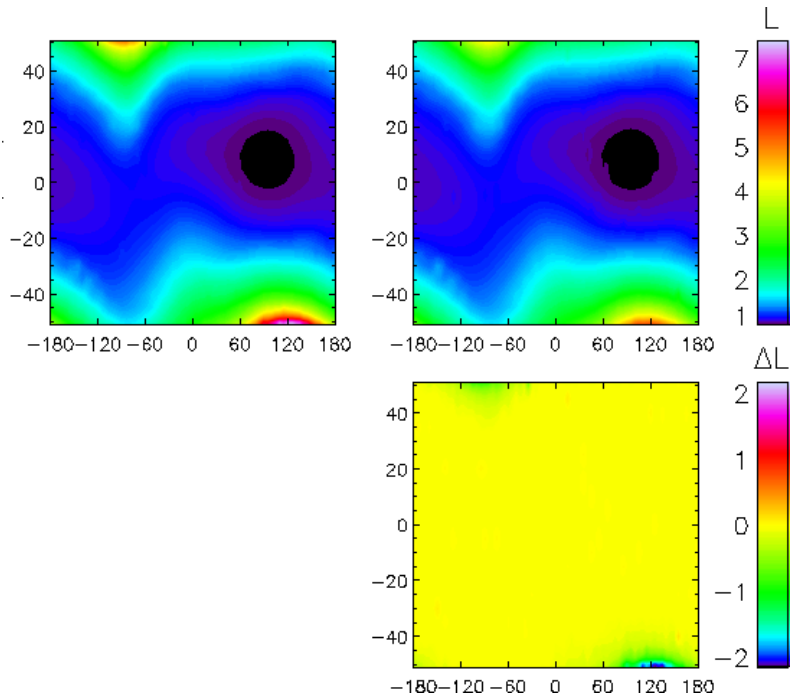

Fig. 5. Comparison between the $L$ parameter calculated with the Tsyganenko89 (upper left panel) and IGRF (upper right panel) models as a function of longitude and latitude. The bottom panel shows the difference between both models.

To check on the validity of this approximation, we plotted the simplified function $f_{1}(\vartheta)=\sin ^{2} \vartheta / 2$ and the full function $f_{2}(\vartheta)=\sin ^{2}(\vartheta) /\left(1+\sqrt{1+\sin ^{3} \vartheta}\right)$ against the co-latitude $\vartheta$, shown in Fig. 4.

The comparison shows the approximation to be valid only in auroral regions, corresponding to a latitude above $\psi \approx 60^{\circ}$ (or co-latitude $\vartheta \approx 30^{\circ}$ ), so that deviations between the derived and the calculated $L$ parameters are to be expected in particular in equatorial regions (cf. Sect. 3).

An example for $L$ parameters derived with the relation by Shea and Smart (1986) by means of numerical simulations with the PLANETOCOSMICS code ${ }^{5}$, a GEANT based simulation code, is shown in Fig. 5. The simulations were carried out for the IGRF model for 2008 as well as for a magnetic field perturbed by the Solar wind according to the Tsyganenko89 model (Tsyganenko, 1989) for the latitudinal range covered by the International Space Station, ISS. Both $L$ parameters show low values in equatorial regions with a minimum around a longitude of about $90^{\circ}$ and values up to $L=6$ in high latitudes. The whole structure shows a strong bend around a longitude of about $-90^{\circ}$. The comparison indicates significant differences between both magnetic field models (bottom panel) only in latitudes of $50^{\circ}$ or higher. If the $L$ parameter is used to interprete data measured close to the Earth's surface it is sufficient to use the IGRF model for the calculations of the $L$ parameter in Sect. 3 .

\footnotetext{
${ }^{5}$ http://cosray.unibe.ch $\sim$ laurent/planetocosmics
}

\section{The McIwain parameter for a realistic planetary magnetic field}

Already for the "second" order approximation of the Earth's magnetic field as a tilted dipole (eccentric dipole approximation), McIlwain's definition cannot be applied, because the magnetic field depends not only on the radius $r$ and the zenith angle (co-latitude) $\vartheta$, but also on the azimuthal angle (longitude) $\varphi$, and the situation becomes even more complicated for a more realistic magnetic field (cf. Stern, 1969). Aside from the difficulties to calculate the $L$ parameter it is still unclear at all, how the $L$ parameter can be defined reasonably for a realistic magnetic field. Therefore, we present below four possible definitions for the $L$ parameter as well as a simple method to calculate them numerically. As mentioned above, we use the IGRF model for 2008 in the following.

The IGRF model uses Eq. (4) with the coefficients $g_{n}^{m}$ and $h_{n}^{m}$ being derived from a network of observations every five years, at last 2005, from which the values for 2008 were extrapolated. These values as well as a numerical code can be accessed via the IGRF website http://www.ngdc.noaa.gov/ IAGA/vmod/igrf.html. In order to calculate the $L$ parameters defined below, we used a simple algorithm to integrate magnetic field lines $\mathbf{r}=\mathbf{r}(s)$ according to the relation

$\mathbf{r}(s+\delta s)=\mathbf{r}(s)+\delta s \frac{\mathbf{B}(\mathbf{r}(s))}{|\mathbf{B}(\mathbf{r}(s))|}$

with the arc length $s$ along the field line and a fixed step size $\delta s$. In order to evaluate the r.h.s., we modified the above mentioned program in such a way that it provides the magnetic field components at the respective point in space. Simultaneously, the following four criteria were evaluated during the integration:

\section{Minimal magnitude of the magnetic field (" $B_{\min }$ ")}

We define the parameter L1 as that distance from the Earth's centre at the point with the minimum magnetic field strength along the magnetic field line. Figure 6 illustrates this definition along an example field line. The colour along the field line gives the magnitude of the magnetic field, the black line points from the centre of the Earth to the point of minimum field strength. Its length, given in Earth radii, gives the value L1.

\section{Passing the geomagnetic equator (" $K=0$ ")}

The second parameter, L2, is defined as the distance at which a magnetic field line crosses the equatorial plane of the tilted dipole field (eccentric dipole) with dipole moment $\mathbf{m}$ and shift $\mathbf{r}_{q}$ as described above. If $\mathbf{r}$ is the vector defining $\mathrm{L} 2$, the scalar product

$$
K=\mathbf{m} \cdot\left(\mathbf{r}-\mathbf{r}_{q}\right)
$$

has to vanish, i.e. $K=0$. This definition is illustrated in Fig. 7. 


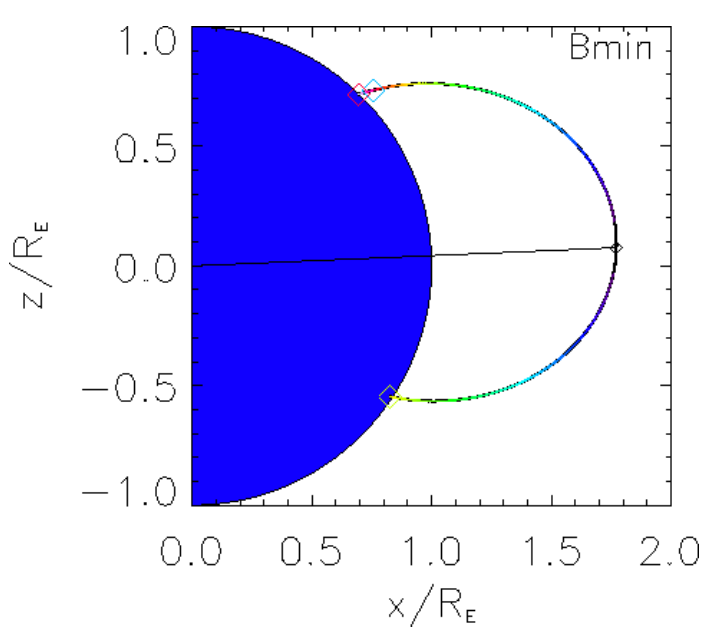

Fig. 6. Illustration of the first definition, $L 1$, for the $L$ parameter along a field line. The colour along the line shows the magnetic field strength. The colour table is the same as in Fig. 5. The red and green diamonds indicate the points, where the field line intersects the surface of the Earth. The field line is identified by the starting point of the integration, marked with a blue diamond.

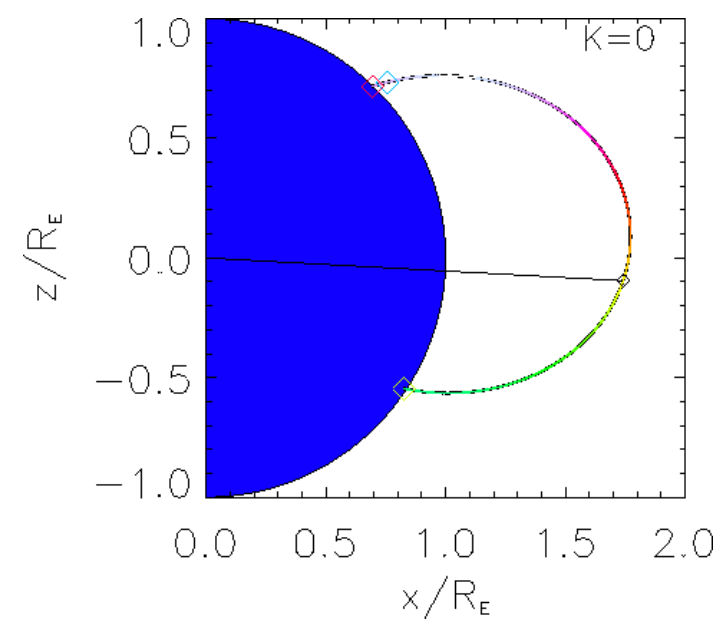

Fig. 7. Same as Fig. 6 but for parameter L2, with the colour now showing the value of $K$. Note that yellow indicates values close to zero.

\section{Maximum distance (" $\mathbf{R}_{\max }$ ")}

Parameter L3, the third possibility, is defined as the maximum distance from the centre of the Earth, which a point along a magnetic field line can attain and is shown in Fig. 8.

\section{Passing the equatorial plane (" $z=0$ ")}

Finally, L4 follows the tradional definition of McIlwain by the passage of a field line through the equatorial plane $z=0$ (cf. Fig. 9).

All four $L$ parameters along the same field line are plotted together in Fig. 10. While the vector defining L4 (" $z=0 ")$

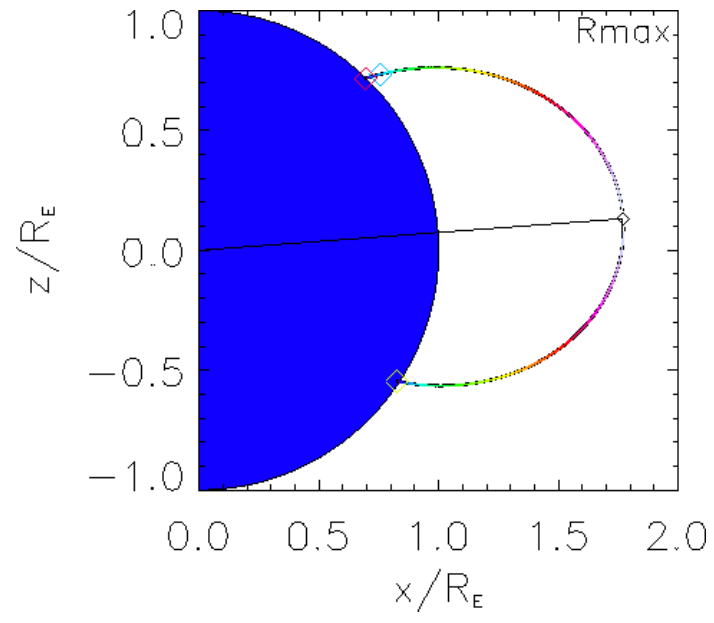

Fig. 8. Same as Fig. 6 but for parameter L3, with the colour now showing the distance $r$.

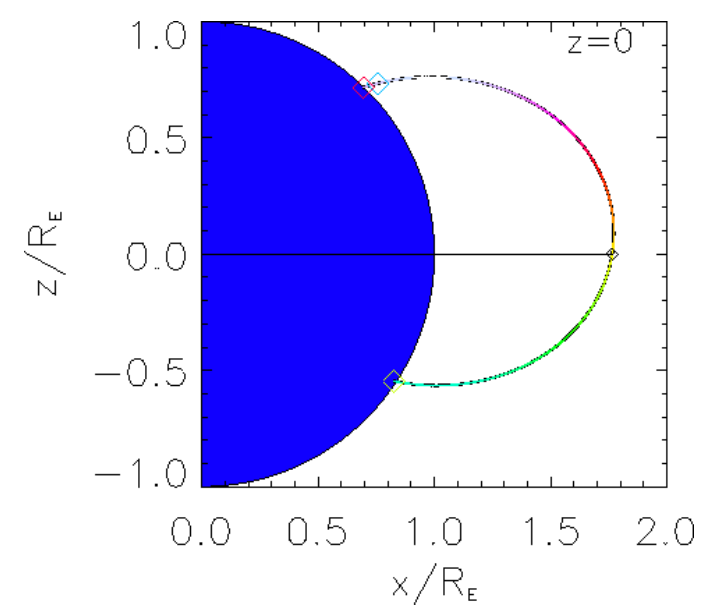

Fig. 9. Same as Fig. 6 but for parameter L4, with the colour now showing the distance of each point of the field line from the equatorial plane.

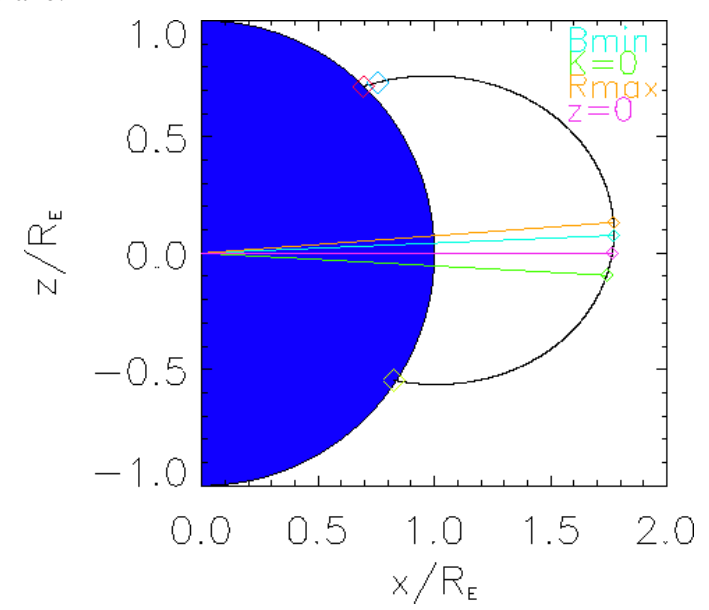

Fig. 10. Illustration of all four $L$ parameters along the same field line. 

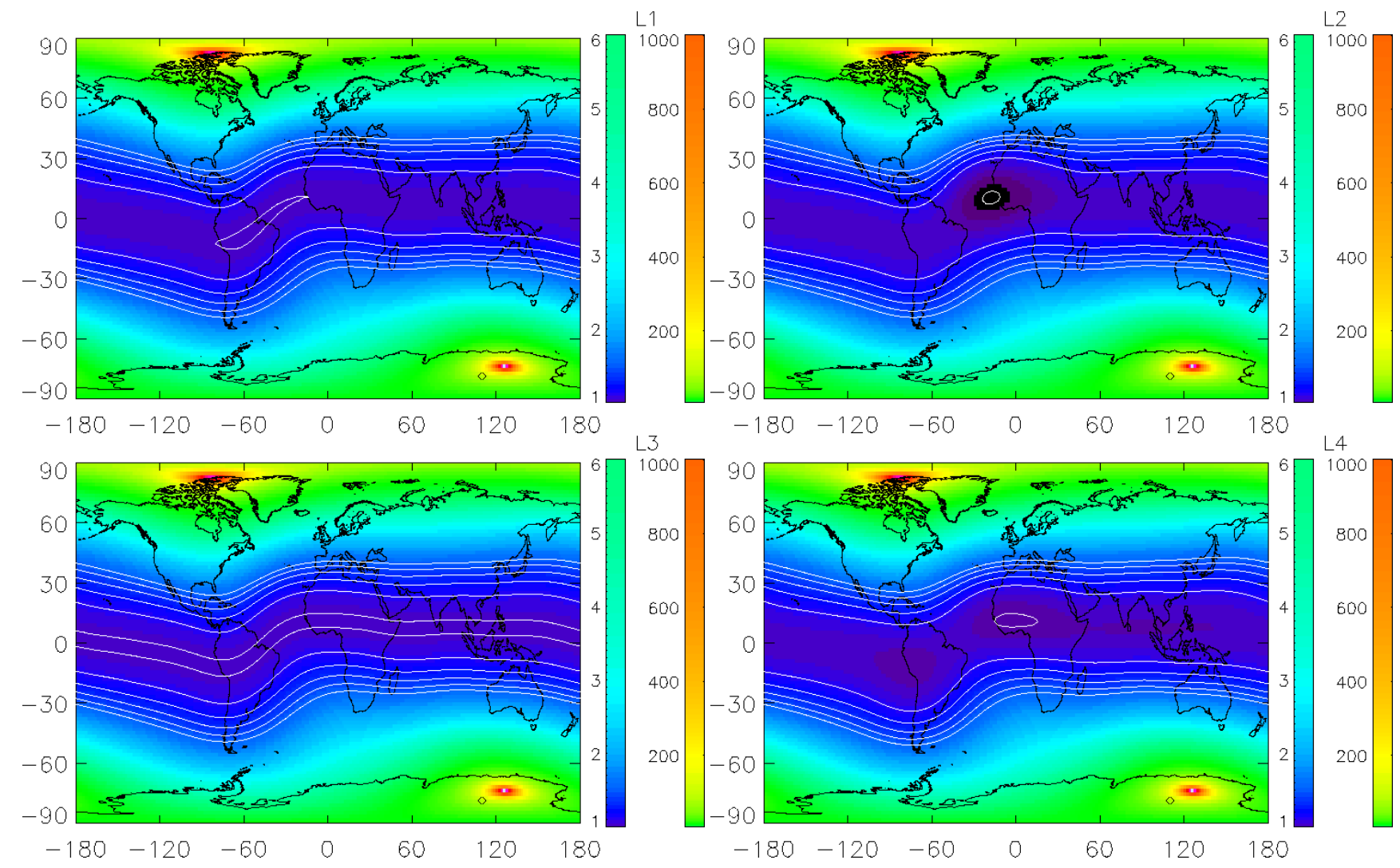

Fig. 11. Mapped $L$ parameters L1 (a), L2 (b), L3 (c) and L4 (d) for the geomagnetic IGRF model for 2008, plotted against the geographical longitude, $\varphi$, and latitude, $\psi=90^{\circ}-\vartheta$. The $L$ parameter reach values of 1 up to 1000 as indicated in the color bars.

lies in the equatorial plane as expected, those for $\mathrm{L} 1$ (" $\mathrm{B}_{\min }$ ") and L3 ("R $\mathrm{R}_{\max }$ ") are located close to each other above the equatorial plane, while that for L2 (" $K=0$ ") lies below it, reflecting the local orientation of the tilted dipole field. Despite of the different locations of these four points, the lengths of the respective vectors and, thus, the $L$ values are quite close to each other.

The results for L1 (a), L2 (b), L3 (c), and L4 (d) are plotted as a function of the geographical longitude and latitude in Fig. 11. In order to capture the large range of $L$ values from the equator towards the poles, the $L$ parameters are shown here in a logarithmic scale.

For a better comparison and discussion of the four suggested $L$ parameters, we plotted the magnetic field components $\mathrm{B}_{r}, \mathrm{~B}_{\varphi}$, and $\mathrm{B}_{\vartheta}$ for the 2008 IGRF model and the tilted dipole in Figs. 12 and 13, respectively.

As already shown in Fig. 10 for a starting point at a latitude around $45^{\circ}$, the $L$ parameter may be defined by different vectors, but their values do not deviate much. This is, however, not the case closer to the equator. Within latitudes of about $\pm 30^{\circ}$, we observe larger differences in the $L$ parameters, while differences beyond this region become less visible. The differences between the various $L$ parameters are caused by the geometry of the IGRF field:
The parameters L1 and L3 hardly diverge from each other. It is interesting to note that their variation with longitude does not follow the geomagnetic equator (cf. Fig. 12a), but with the curve $B_{r}=0$, i.e. the yellow line in Fig. 12a. Larger deviations can be seen for L2, in particular, with a minimum above West Africa coinciding with the region, where the radial component, $B_{r}$, shows the largest differences between the IGRF field and the tilted dipole (cf. Fig. 12a for the IGRF model and Fig. 13a for the tilted dipole). For parameter L4 one can recognise the variation of the IGRF field around the equatorial plane $z=0$ by structures above West Africa and South America (cf. Fig. 11d).

From these observations, we may conclude that L2 and L4 reflect different magnetic field models (tilted and aligned dipoles, respectively) rather than the geometry of the actual magnetic field, leaving us with only two parameters, L1 and L3. Since for most applications the $L$ parameter is used to identify the origin of particles, e.g. from the radiation belts, we choose L3 as the most applicable parameter, because it contains "geometrical" informations about the respective field line. Regarding the magnitude of the magnetic field $|\mathbf{B}|$ we, moreover, want to point out that the South Atlantic Anomaly (SAA), the region of a very low magnetic field above South America, is visible only in the IGRF field, 

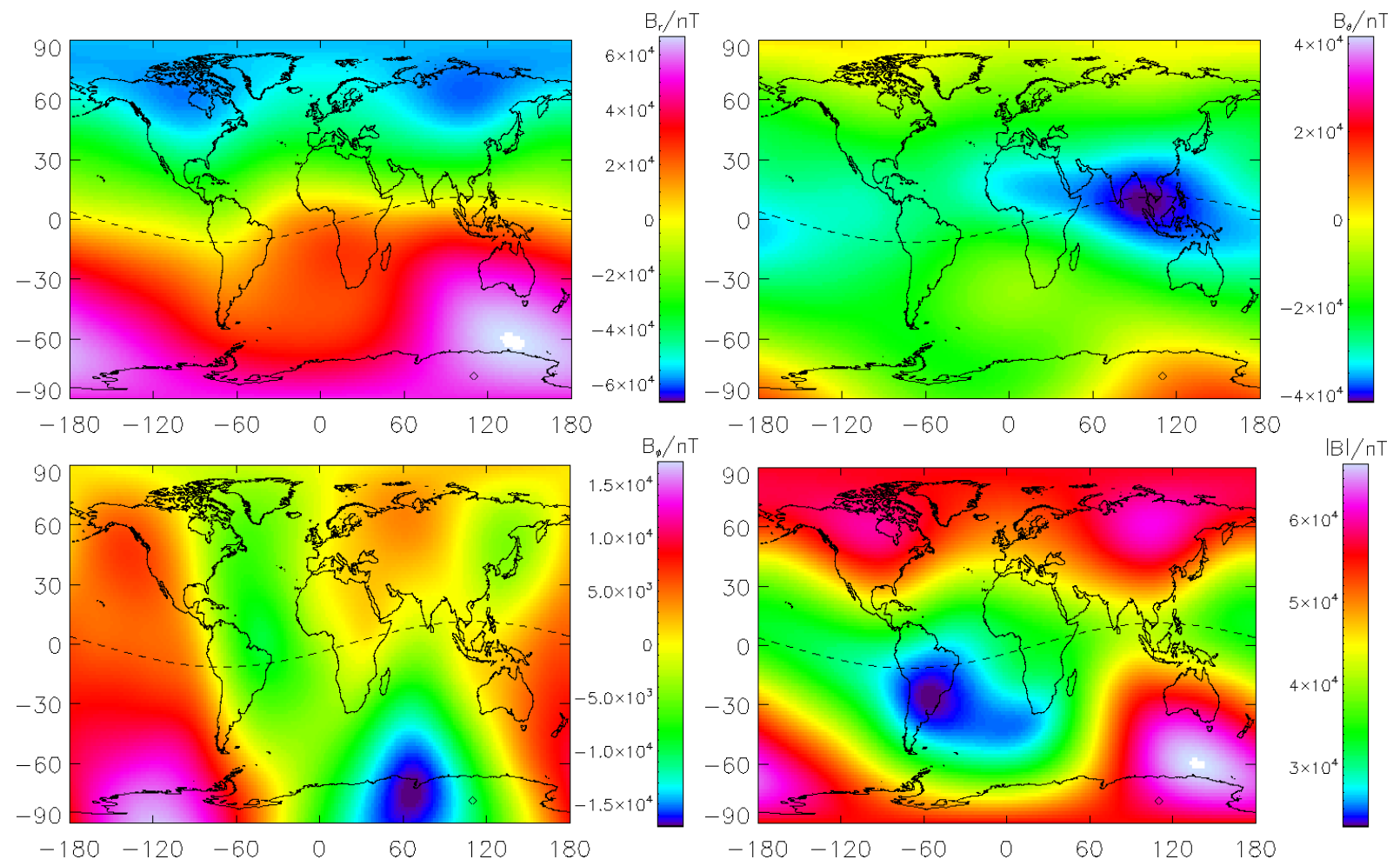

Fig. 12. IGRF field of the Earth in 2008 with the magnetic components $\mathrm{B}_{r}(\mathbf{a}), \mathrm{B}_{\vartheta}(\mathbf{b})$, and $\mathrm{B}_{\varphi}$ (c) as well as the magnitude $|B|(\mathbf{d})$. The dashed line shows the magnetic equator of the tilted dipole field.
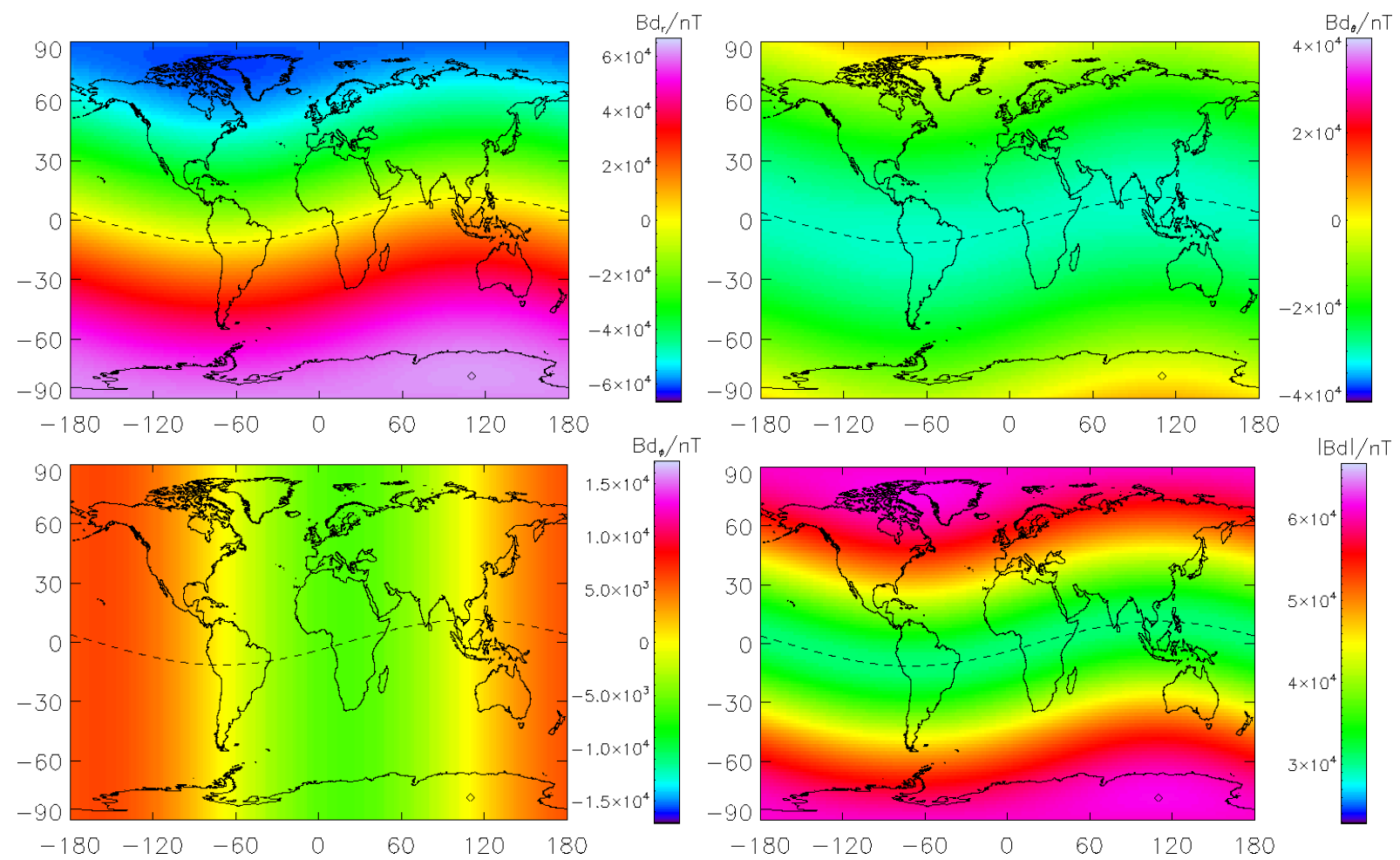

Fig. 13. Same as Fig. 12, but for the tilted dipole field (geocentric dipole approximation). 
but not in the tilted dipole, a fact already observed in calculations by Roederer et al. (1967), who showed that the SAA is caused by higher $(n \geq 2)$ terms of the expansion (4) rather than by the shift of the tilted dipole out of the centre of the Earth.

Comparing our new method with the traditional described in Sect. 2 and illustrated in Fig. 5 shows in general a quite similar structure, but also large deviations in equatorial regions, in particular that of very low values for $L$ above India, which can be seen in none of the new definitions of the $L$ parameter shown in Fig. 11. Because the values in Fig. 5 were obtained with simulated rigidities via the relation by Shea and Smart (1986), this deviation may be caused by the approximation (15) which is not valid close to the equator as well as by the very high rigidities corresponding to the low $L$ parameters.

In order to understand the last point, we consider the $r$ and $\vartheta$ components of the IGRF model, shown in Fig. 12a and b. We find a maximum in $B_{\vartheta}$, which is located actually above India, whereas $B_{r}$ takes very low values there. With respect to the rigidities, this means that particles hitting the Earth's surface in radial direction feel a magnetic field essentially perpendicular to their direction of motion, allowing only very energetic particles to reach the surface, leading to the observed high values for the cut-off rigidities. The latter, however, merely reflect local field structures, but do not provide the required informations about the global one. Moreover, the rigidities do not fully take into account the other field components in this region as the direct computation does. Note, that also the feature of the magnetic field above India cannot be seen in the tilted dipole field (cf. Fig. 13).

Furthermore, we show in Fig. 14 the relative differences between each $L$ parameter relating to the $\mathrm{L} 3$ parameter. The parameters L1 and L3 hardly diverge from each other (cf. Fig. 14a), whereas the deviation for L2 (b) and L4 (c) are mostly noticeable in the equatorial plane with a slightly difference above India for L2. On the contrary, the deviation between L3 and the traditional $L$ parameter computed with the rigidities (d) is strong and varies for all latitudes, with the highest variation being $15 \%$.

We may conclude that the traditional method does not in all regions reflect the magnetic field geometry, because it uses the indirect way via cut-off rigidities. Furthermore, the relation by Shea and Smart (1986), Eq. (17), is based on Størmer orbits performed for an aligned dipole. In particular, the Eq. (15) is valid only in higher latitudes (cf. Fig. 4). A further point is the fact that the values for $\tilde{K}$ and $q$ derived by Shea and Smart (1986) are based on data from the early $80 \mathrm{~s}$ and thus cannot reflect the temporal evolution of the magnetic field. Moreover, the new method allows to compute the $L$ parameter along a certain orbit of a spacecraft for different altitudes. In addition, providing the cut-off rigidities with numerical methods or data is not only time-consuming, but also difficult for different altitudes and considerably more inaccurate, because a mesh with limited grid size has to be used.
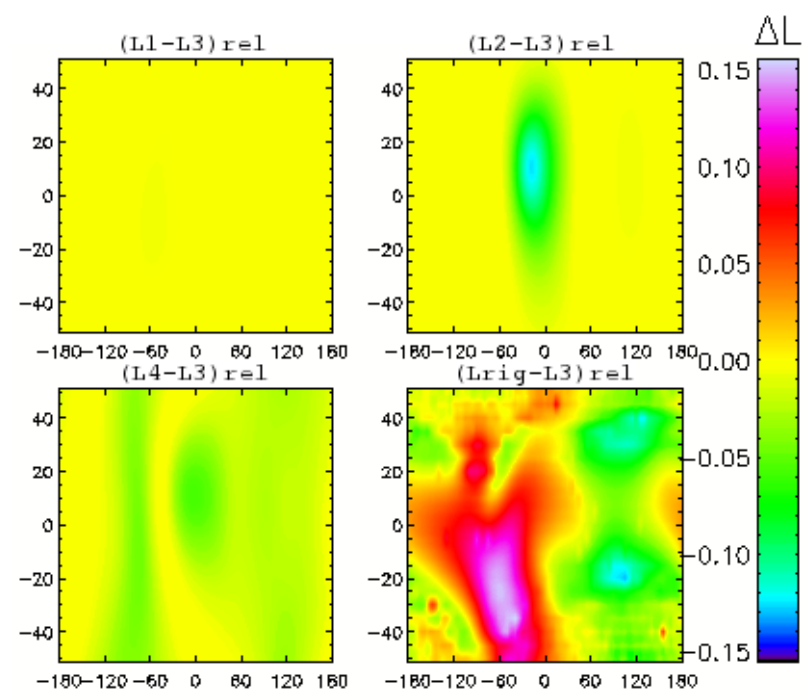

Fig. 14. Relative difference between the L3 parameter and L1 (a), L2 (b), L4 (c) and the traditional $L$ parameter computed indirectly with the rigidities $(\mathbf{d})$.

\section{Summary and conclusions}

An important quantity to identify the origin of particles in planetary magnetic fields is the $L$ parameter, which so far has only been defined for an aligned dipole field and specifies the distance of a field line from the Earth's centre in the equatorial plane measured in Earth's radii. The latter was at first defined by McIlwain (1961) and thus is called the Mcllwain parameter, which at a given co-latitude $\vartheta_{0}$ is given by the simple relation $L=1 /\left(\sin ^{2} \vartheta_{0}\right)$. Up to now the $L$ parameter could only be assessed indirectly by employing cut-off rigidities, $P$, of energetic particles, taken e.g. from numerical simulations, which subsequently are related to the respective $L$ parameter by means of the Equation $P=\tilde{K} / L^{q}$. Such a relation was originially found by Størmer (1955) only for regions in high latitudes in an aligned dipole field. A more accurate relation was later derived by by Shea and Smart (1986). Therefore, not only a newer and direct method is neccessary to calculate the $L$ parameter, but a general significant definition for the $L$ parameter is desirable as well. We successfully found a simple method, as well as four potential definitions of the $L$ parameter for a realistic magnetic field described as:

1. $\mathrm{L} 1$ is defined as the distance from Earth's centre at the point with the minimum magnetic field strength.

2. L2 describes the distance at which a magnetic field line crosses the equatorial plane of the tilted dipole field with the dipole moment $\mathbf{m}$ and shift $\mathbf{r}_{q}$.

3. The parameter L3 defines the point along a field line with the maximum distance from the centre of Earth. 
4. L4 follows the traditional definition of the McIlwain by the passage of a field line through the equatorial plane $z=0$.

Since L2 and L4 rely on simplified magnetic field models (tilted and aligned dipole fields, respectively), but do not reflect the geometry of the actual magnetic field, the parameters L1 and L3 appear to be the more appropriate definitions. From those we choose L3 as the most applicable definition for a realistic magnetic field, because compared to L1 it contains information about the excursion of the respective field line.

Comparing the traditional method with our new method we recognise large deviations, mostly in equatorial regions, that are caused at first by the use of the cut-off rigidities and second by simpifying, but at least partially inaccurate approximations leading to the relation $P=\tilde{K} / L^{q}$. By employing the latter relation to obtain the $L$ parameter by the traditional, quite cumbersome method, only parts of the geomagnetic structure can be reflected compared to the new, direct computation that provides required information about the global geomagnetic structure.

The defintion of the $L$ parameter as the maximum distance along a magnetic field line and the method to calculate it allows for the first time to describe charged particles linked to magnetic field lines in an actual and realistic planetary magnetic field, in particular also for other planets in the Solar System.
Acknowledgements. This paper was partially financially supported by the Deutsche Forschungsgesellschaft, DFG, via the project CAWSES (He 3279/8-2 and 8-3).

Edited by: H.-J. Fahr

Reviewed by: two anonymous referees

\section{References}

Connerney, J.: Magnetic fields of the outer planets, J. Geophys. Res., 18, 659-679, 1993.

Gauss, C. F.: Allgemeine Theorie des Erdmagnetismus: Resultate aus den Beobachtungen des magnetischen Vereins im Jahre 1836, Göttinger Magnetischer Verein, Leipzig, 1-52, 1836.

McIlwain, C. E.: Coordinates for Mapping the Distrubution of Magnetically Trapped Particles, J. Geophys. Res., 66, 3681-3691, 1961.

Roederer, J., Welch, J., and Herod, J.: Longitude dependence of geomagnetically trapped electrons, J. Geophys. Res., 72, 44314447, 1967.

Shea, M. and Smart, D.: Estimating cosmic ray vertical cutoff rigidities as a function of the McIlwain parameter for different epochs of the geomagnetic field, Phys. Earth Planet. Interiors, 48, 200-205, 1986.

Stern, D. P.: Euler Potentials, Am. J. Phys., 4, 494-501, 1969.

Størmer, C.: Polar Aurora, Clarendon Press Oxford, 294 pp., 1955.

Tsyganenko, N.: A magnetospheric magnetic field model with a warped tail current sheet, Planet. Space Sci., 37, 5-20, 1989. 